\title{
Pivô Semântico, Tópico e Foco em Avá-Canoeiro do Tocantins (família Tupí-Guaraní)
}

\author{
Ariel Pheula do Couto e Silva ${ }^{1}$
}

\begin{abstract}
Resumo
Este artigo trata de três elementos da sintaxe da variedade Avá-Canoeiro do Rio Tocantins (doravante Av.C-T) (família Tupí-Guaraní), a saber, as categorias de pivô-semântico (SmP), de tópico e de foco. Argumentamos que para as línguas da família Tupí-Guaraní que se assemelham ao Av.C-T, o conceito de pivô semântico é fundamental para a compreensão do engatilhamento de marcações de correferencialidade, o qual situa-se na interface entre a sintaxe e a semântica. O tópico, em Avá-Canoeiro, se expressa por meio da marcação de argumentos S/A por meio de prefixos pessoais, em orações no Modo Indicativo I; enquanto, no Modo Indicativo II, é um elemento circunstancial que passa a funcionar como tópico. Diferentemente da variedade Avá-Canoeiro do Rio Araguaia, o Av.C-T desenvolveu uma partícula de foco tõ, a qual marca tanto os argumentos S/A e expressões adverbiais quanto predicados, ocorrendo sempre em primeira posição na sentença.
\end{abstract}

Palavras-chave: família Tupí-Guaraní. Avá-Canoeiro. Pivô semântico. Tópico. Foco.

\begin{abstract}
This study deals with three elements of the Tocantins River variety os the Avá-Canoeiro language syntax (hereinafter Av.C-T) (Tupí-Guaraní family), namely the semantic pivot $(\mathrm{SmP})$, topic and focus categories. We argue that in most Tupí-Guaraní family languages, the concept of semantic pivot is fundamental to understand switch reference, which in these languages depends on the interface between syntax and semantics. The topic in AváCanoeiro is expressed by the marking $\mathrm{S} / \mathrm{A}$ arguments in sentences in the Indicative I mode, by means of personal prefixes; while in the Indicative II mode it is a circumstantial element that function as topic. Unlike the Avá-Canoeiro variety of the Araguaia River, the Av.C-T developed a focus particle tõ, which marks both the S / A arguments, as well as adverbial and predicate expressions, always occurring in the first position of the sentence.
\end{abstract}

Keywords: Tupí-Guaraní Family. Avá-Canoeiro. Semantic pivot. Topic. Focus.

\section{Introdução}

Tratamos, neste artigo, de três elementos da sintaxe $\mathrm{da}^{2}$, variedade do Rio Tocantins da língua Avá-Canoeiro (doravante Av.C-T), a saber: pivô semântico $(\mathrm{SmP})$, tópico e foco.

\footnotetext{
${ }^{1}$ Doutorando em Linguística na Universidade de Brasília. Bolsista CAPES.

${ }^{2}$ Este estudo é uma versão revisada de minha dissertação de mestrado intitulada fonologia, morfossintaxe e sintaxe da língua Avá-Canoeiro do Tocantins".
} 
A língua Avá-Canoeiro possui, na atualidade, duas variedades diatópicas: o Avá-Canoeiro do Araguaia (doravante Av.C-A), cujos falantes se localizam majoritariamente na Ilha do Bananal, sul do estado de Tocantins; e o AváCanoeiro do Tocantins (Av.C-T), cujos falantes se localizam na Terra Indígena Avá-Canoeiro, ao norte do estado de Goiás. Essa língua é classificada como pertencente ao subramo IV da família Tupí-Guaraní, tronco Tupí (Rodrigues, 1985; Rodrigues \& Cabral, 2002), juntamente com as línguas Tapirapé, Asuriní do Tocantins, Parakanã, Suruí (Mujetire), Tembé, Guajajára e Turiwára† †

A seguir, discutimos acerca da expressão de pivô semântico em Av.C-T.

\section{A expressão pivô semântico (PSm) em Avá-Canoeiro do Tocantins}

Foley e Van Valin (1984: 108-124) distinguem dois tipos de pivôs nas línguas do mundo, os quais são fundamentais, por exemplo, para a marcação de correferencialidade: o pivô pragmático e o pivô semântico.

O pivô pragmático ( $\mathrm{PrP}$ ), segundo o autor (op. cit.) ocorreria em línguas em que o sujeito ocupa uma posição de relevo, com possibilidade de passivização, no caso de línguas nominativo-acusativas, como o Inglês e o Português; e de antipassivização, no caso de línguas ergativo-absolutivas, como o Dyirbal.

Em Inglês, nas orações ativas, enquanto o argumento sintático S/(A) codifica o papel de agente (Actor), o argumento O(bjeto), de verbos transitivos, codifica o papel de paciente (Undergoer). Já nas orações passivas, o argumento S(ujeito) codifica o papel de paciente, enquanto o agente é expresso, de forma facultativa, por meio de um sintagma preposicional. Os exemplos abaixo são dados por Foley e Van Valin (1984: 108):
(a) The boy hit the ball 'o garoto chutou/bateu na bola'
(b) The ball was hit (by the boy) 'a bola foi chutada/batida (pelo garoto)'

Consoante os autores, em Dyrbal, o papel de Actor seria expresso tanto em orações ativas, por meio de S/A, quanto em orações passivas, por meio de S. Nestas últimas, o Undergoer vem expresso por meio de um sintagma preposicional ou marcado com o caso dativo, como mostram os exemplos seguintes (Foley e Van Valin, 1984: 111):

\footnotetext{
${ }^{3}$ Seguimos, neste artigo, a análise dos macro papéis semânticos de Actor e Undergoer, importantes para o conceito de pivô (Foley e Van Valin 1984). No entanto, ao tratarmos de argumentos sintáticos, seguimos Dixon (1994) para a distinção de S (primeiro argumento de verbo intransitivo); A (primeiro argumento de verbo transitivo); e O (segundo argumento de verbo transitivo).
} 
(4.10) (a) Balan dugumbil bayul yara-ngu bura-n mulher-ABS(U) homem-ERG(A) ver-TNS 'O homem viu a mulher'
(b) Bayi yara bagun dugumbil-gu bural-na-nu homem-ABS(A) mulher-DAT(U) ver-ANTI-TNS 'o homem viu a mulher'

Para os autores (op. cit.: 114), a escolha do argumento para funcionar como pivô, nestas situações, não é determinada por bases semânticas, uma vez que na maioria dos verbos transitivos em Inglês e em Dyirbal, tanto o Actor (agente) quanto o Undergoer (paciente) podem funcionar como pivô. Com isso, a escolha seria influenciada ou por fatores discursivos, como referência alternada, como nos exemplos (4.9) e (4.13)) ou por topicalidade, como nos exemplos (4.7) e $(4.8))^{4}$ (Foley e Van Valin, 1984: 109-113).

(4.9) (a) Oscar went to the store and spoke to Bill.

'Oscar foi a loja e chamou Bill'

(b) * Oscar went to the store and Bill spoke to (him)

'Oscar foi a loja e Bill chamou (o)'

(c) Oscar went to the store and was spoken to by Bill.

'Oscar foi a loja e foi chamado por Bill'

(4.13) (a) Balan dugumbil bani-nu banul yaaa-ngu bura-n mulher-ABS(A) vir-TNS homem-ERG(A) ver-TNS 'A mulher veio e o homem (a) viu'

(b) *Bayi yara bani-nu bagun dugumbil bura-n homem-ABS(A) vir-TNS mulher-ABS(U) ver-TNS 'o homem veio e viu a mulher'

(c) Bayi yara bani-nu bagun dugumbil-gu bural-na-nu homem-ABS(A) vir-TNS mulher-DAT(U) ver-ANTI-TNS 'O homem veio e viu a mulher'

(4.7) (a) It seems that Paul caught the wombat ("Parece que Paulo pegou o vombate").

(b) Paul seems to have caught the wombat ("Paule parece ter pego o vombate").

\footnotetext{
${ }^{4}$ Tomamos a liberdade de traduzir as glosas para o português bem como adaptar os exemplos encontrados em (4.9), substituindo 'falar' por 'chamar'. Em (4.7) e (4.8), fornecemos a tradução para os exemplos originais em inglês.
} 
(c) *The wombat seems Paul to have caught $\left({ }^{*}\right.$ "O vombate parece Paulo ter pego").

(d) The wombat seems to have been caught by Paul ("O vombate parece ter sido pego por Paulo).

(4.8) (a) John expects that Paul will catch the wombat ("João espera que Paulo pegará o vombate").

(b) John expects Paul to catch the wombat ("João espera Paulo para pegar o vombate").

(c) *John expects the wombat Paul catch (*"João espera que o vombate Paulo pegue”).

(d) John expects the wombat to be caught by Paul ("João espera que o vombate seja pego por Paulo").

O segundo tipo de pivô trabalhado pelos autores (op. cit.: 115-124) é chamado de pivô semântico (SmP) e se caracteriza, em línguas como o Choctaw, pelas "[...] considerações acerca de topicalidade e discurso não interferirem na seleção do pivô, que é realizada inteiramente no nível semântico e lexical" (Foley \& Van Valin 1984: 117). Em Choctaw, há uma hierarquia semântica (agente $>$ paciente $>$ dativo) que determina a escolha do argumento a ser marcado com at 'pivô', conforme observado nos exemplos em (4.14) (Foley e Van Valin 1984: 115).
(a) Hattak at $\varnothing$-iya-h
homem PVT 3A-ir-PRES
'O homem vai'
(b) Hattak at $\varnothing$-abika-h
homem PVT 3U-doente-PRES
'O homem está doente'
(c) Hattak at oho:yoh (ã:) Ø-Ø-pi:sa-h
homem PVT mulher OBL 3A-3U-ver-PRES
'O homem vê a mulher'
(d) Hattak at oho:yoh (ã:) i:-Ø-nokšo:pa-h homem PVT mulher OBL 3DAT-3U-medo-PRES
'O homem está com medo da mulher'

Segundo os autores (op. cit.), o status do SmP é aparente no monitoramento do sistema de referência alternada existente na língua, conforme ilustrado pelos exemplos contidos em (4.15) (Foley e Van Valin, 1984: 116), que reproduzimos abaixo: 

(a) Ø-Ø-pissa-ča: Ø-iya-h
3A-3U-ver-MESMO.PVT 3A-ir-PRES

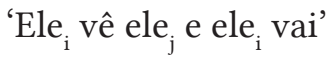
(b) Ø-Ø-pisa-na: $\quad$-iya-h
3A-3U-ver-DIFF.PVT 3A-ir-PRES
'Ele ${ }_{i}$ vê ele ${ }_{j}$ e ele ${ }_{j / k}$ vai'

Para Foley e Van Valin (op. cit.: 117), o tipo mais comum de SmP nas línguas do mundo é aquele que agrupa o argumento Agente de verbos transitivos com o único argumento de verbos intransitivos, isto é, agrupando $\mathrm{A}$ e $\mathrm{S}$, segundo a terminologia de Dixon (1979 apud Foley e Van Valin, op. cit.).

A partir da distinção estabelecida pelos autores entre PrP e SmP, observamos que o Av.C-T se diferencia das línguas que possuem pivô pragmático, como o Inglês e o Dyirbal, por não possuir estruturas na voz passiva e na voz antipassiva, aproximando-se então de línguas que possuem SmP, uma vez que o argumento que aciona a marcação de correferencialidade não precisa estar expresso sintaticamente ou marcado por meio de prefixos no núcleo do predicado. Desta forma, argumentamos que se trata de um tipo de pivô semântico, mas não no nível semântico-lexical, como descrito para o Choktaw (cf. Foley \& Van Valin 1984: 115-118), e sim no nível semântico-sintático.

Em Avá-Canoeiro, tanto nomes como verbos podem funcionar como argumentos ou predicados. Nomes marcados pelo caso argumentativo podem funcionar como argumento $\mathrm{S} / \mathrm{A} / \mathrm{O}$ de um predicado, como objeto de posposição, como determinante de outro nome; e quando marcados por casos locativos, funcionam como sintagmas adverbiais. Já os nomes sem marcação de caso funcionam como núcleo de predicados intransitivos possessivos ou existenciais, sendo seu argumento $\mathrm{S}$ sinalizado no núcleo por meio de prefixo relacional.

Os verbos, classe que se distingue por se combinar com prefixos pessoais, ao serem marcados por sufixo de caso argumentativo, sem prefixos pessoais, funcionam como argumento, em contextos semelhantes ao de nomes marcados pelo mesmo sufixo do caso argumentativo; e ao serem marcados por casos locativos, funcionam como sintagmas adverbiais.

Uma vez que tanto nomes quanto verbos podem predicar, muito provavelmente seria a categoria de predicado ao invés da classe lexical dos verbos e nomes, a que estaria relacionada a expressão de pivô.

Para Cabral et al. (2010: 102), o pivô nas línguas Tupí-Guaraní é o argumento S/A da oração principal, o qual aciona a marcação de correferencialidade em orações dependentes. Neste sentido, segundo os autores, "a orientação da correferência em Tupí-Guaraní é o Actor (que corresponde ao sujeito de verbos transitivos e sujeito de verbos intransitivos), enquanto que o alvo é o Undergoer (que corresponde ao objeto e ao sujeito de construções dependentes)". 
Argumentamos, a seguir, que em línguas Tupí-Guaraní como o Av.C-T e o Asuriní do Tocantins (subramo IV) e Apiaká (subramo VI), o pivô não diz respeito (a) aos argumentos $\mathrm{S} / \mathrm{A}$ expressos sintaticamente; (b) ao argumento marcado ou sinalizado no núcleo do predicado, respectivamente por meio de prefixos pessoais ou prefixos relacionais; nem (b) ao tópico ou foco da oração (o que será tratado nas seções seguintes). O pivô, nessas línguas, relacionase à semântica do predicado, em nível sintático-semântico, e não em nível semântico-lexical.

Apresentamos, em seguida, alguns exemplos para ilustrar a diferença entre os argumentos S ou A marcados no núcleo do predicado e a expressão de SmP. No exemplo (1) o S é marcado na oração principal por meio de prefixo pessoal no núcleo do predicado verbal 'ir', que se encontra modo Indicativo I, o qual é correferente com o argumento S do predicado do verbo 'caminhar' da oração dependente, no modo Gerúndio, sinalizado no núcleo do predicado por meio de prefixo relacional correferencial $\left(\mathrm{R}^{3}\right)$.

$$
\begin{aligned}
& \text { S-P } \quad \mathbf{R}^{3}-\mathbf{P} \\
& \text { t a-a we-ata-w } \\
& \text { PROP 1-ir 1CORR-caminhar-GER } \\
& \text { 'é para eu ir caminhar (amanhã)' }
\end{aligned}
$$

A partir deste exemplo isolado, pode-se interpretar o prefixo pessoal da Série I, em 'ir', como a expressão do pivô nesta língua, acionando a marcação de correferencialidade na oração dependente.

No entanto, as orações no modo indicativo II mostram que, mesmo com S/O não codificados no núcleo verbal por meio de prefixos pessoais, o pivô contido na semântica do predicado aciona a marcação de correferencialidade em uma oração dependente. No exemplo (2), a oração no modo gerúndio, por possuir também valor adverbial, aciona o modo indicativo II ao ocorrer anteposto à oração principal. Neste caso, 'puxar' é núcleo de um predicado transitivo e tem nele sinalizado a não contiguidade sintática do $\mathrm{O}$, por meio de prefixo relacional 2. O SmP, neste sentido, não se refere a uma marcação no núcleo do predicado da oração principal no modo indicativo II.
(2) $\mathbf{R}^{3}-\mathbf{P}$
$\mathbf{R}^{2}-\mathbf{P}$
O
ae tõ
ow-ев-а
i-t $\int 0-w$
a
DÊIT FOC 3CORR-C.C.-ir+GER $\mathrm{R}^{2}$-puxar-IND.II DÊIT
'ele, para levá-lo consigo, puxou-o, este' 
No exemplo (3) seguinte da língua Asuriní do Tocantins, temos um contexto semelhante ao que ocorre com o exemplo (1) do Av.C-T. O argumento $\mathrm{S}$ de 'dançar' marcado no núcleo do predicado da oração principal, no modo indicativo I, é correferente com o S de 'vir'.

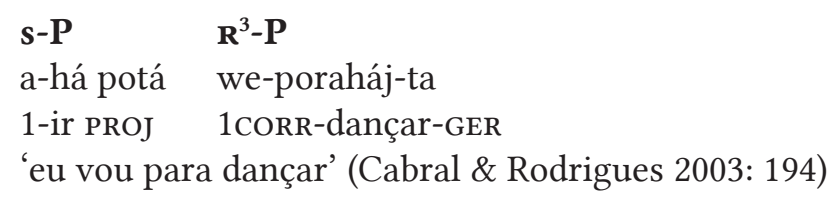

No entanto, conforme observado no exemplo (4), apresentado em seguida, um predicado no modo indicativo II, sem a marcação de prefixos pessoais, aciona marcação de correferencialidade no núcleo verbal 'pegar'. Nesse exemplo, o A do predicado 'pegar' é correferente com o S de 'ir'. Por se tratar de um verbo intransitivo, somente o argumento $\mathrm{O}$ é sinalizado núcleo do predicado no modo gerúndio, por meio do prefixo relacional 2.

(4) SP

$\begin{array}{ll}\text { Paranó-a } & \text { r-opí } \\ \text { rio-ARG } & \mathrm{R}^{1} \text {-com }\end{array}$

$$
\mathbf{R}^{2}-\mathbf{P} \quad \mathbf{O} \quad \mathbf{R}^{2}-\mathbf{P}
$$$$
\text { i-há-j }
$$

ipirá- $\varnothing$

'ele foi pelo rio para pegar peixe' (Cabral \& Rodrigues 2003: 88)

Em predicados nominais de orações existenciais, o seu argumento S é sinalizado no núcleo por meio de prefixo relacional. E, caso o seu determinante não esteja contíguo ao predicado, isto é, imediatamente à esquerda, formando com este um constituinte sintático, este será sinalizado no núcleo por meio de prefixo relacional 2, uma vez que pode já ter sido ou ainda será enunciado pelo falante. No exemplo (5) da língua Apiaká, o pivô 'eu' da oração principal 'existe raiva de mim', ocorre na semântica do predicado existencial, não sendo expresso por marcas pessoais. No entanto, ele engatilha a marcação de correferencialidade na oração dependente posposta, no modo gerúndio.

$$
\begin{array}{lll}
\mathbf{R}^{2}-\mathbf{P} & \mathbf{S} & \mathbf{R}^{3}-\mathbf{P} \\
\varnothing \text {-mandarahym } & \text { si } & \text { te-ko- } \beta \text { o } \\
\mathrm{R}^{2} \text {-raiva } & 1 & \text { 1CORR-estar.em.movimento-GER } \\
\text { 'existe raiva de mim, eu estando em movimento' (Sousa 2017, em preparação) }
\end{array}
$$

É importante observar, com respeito ao prefixo pessoal utilizado quando 1(2(3)) age sobre 2(3), que, mesmo correspondendo a um argumento O, este não se refere ao pivô $\mathrm{SmP}$, diferentemente de línguas Túpí-Guaraní como o Asuriní do Tocantins, em que (cf. Cabral et al, 2010). No exemplo (6) do Asuriní do Tocantins, o O ( $\left.2^{\mathrm{a}} \mathrm{p}\right)$ é marcado no núcleo do predicado do verbo -owaesãng 'olhar na face', 'encarar'. No entanto, o SmP, que correspondente ao argumento A do predicado, não é expresso sintaticamente ou morfossintaticamente no 
predicado, mas aciona correferencialidade na oração dependente com o verbo posicional 'estar sentado', conforme observado no exemplo a(6).

\author{
(6) o-P $\quad \mathbf{R}^{3}-\mathbf{P}$ \\ oro-owaesáng wet-oín-a \\ 2O-olhar.na.face/encarar 1CORR-estar.sentado-GER \\ 'eu estou de encarando você' (Cabral \& Rodrigues 2003: 172)
}

Desta forma, observamos que nessas línguas o $\mathrm{SmP}$, fundamental para compreender as estruturas de correferencialidade, faz parte da semântica do predicado, estando então na interface entre a semântica e a sintaxe, e não na semântica do nome ou do verbo, em termos semântico-lexicais.

Na subseção seguinte descrevemos a expressão de tópico em línguas como o Av.C-T e Asuriní do Tocantins, uma vez que esta categoria é mais relevante para essas línguas do que a noção propriamente dita de sujeito. Para tanto, diferenciamos os argumentos marcados no núcleo do predicado dos argumento sintáticos, os quais são sintaticamente facultativos, mas obrigatórios no nível do discurso. Contrastamos, desta forma, o que é tópico nessas línguas do conceito trabalhado anteriormente de pivô semântico ${ }^{5}$.

\title{
Tópico em Avá Canoeiro do Tocantins
}

Vieira (2015: 669), referindo-se às línguas Tupí-Guaraní, ressalta que "identificar tópico e foco em línguas com ordem livre não é uma tarefa das mais simples, uma vez que parece impossível determinar a localização dos constituintes na arquitetura funcional da oração".

Payne (1994: 316) comenta acerca da hierarquia de pessoa $(1>2>3)$ encontrada em línguas Tupí-Guaraní e a relação dessa hierarquia com a expressão de topicalidade (cf. Payne op. cit.; Monserrat \& Facó Soares 1983):

Because 1st and 2nd person participants are already, simply by the pragmatics of the speech act, individuated from the world of things « out there » to be talked about, they are inherently more topical than 3rd persons. The speech act participants are also always available in memory; by definition, if a hearer is attending to a speaker, the hearer must always have an "open file" for the speaker. There is also natural sense in which speech act participants are generally taken for granted as "more important" or the "natural center of interest", over 3rd persons. Thus, regardless of any particular discourse context, the hierarchy (...) can be taken as an inherent topicality hierarchy. ${ }^{6}$

\footnotetext{
${ }^{5}$ Para uma diferenciação entre a expressão dos conceitos de pivô pragmático $(\mathrm{PrP})$ e tópico nas línguas do mundo, vide Foley e Van Valin (1984: 124-134).

6 "Por conta das $1^{\text {as }}$ e $2^{\text {as }}$ pessoas participantes serem, simplesmente por conta do ato pragmático, individuadas do mundo das coisas "de fora" acerca do qual se fala, elas são 
Em Av.C-T, observamos, consoante Borges (2006: 158-161), que também há hierarquia de referência de pessoa $(1>2>3)$, sendo somente um argumento marcado ou sinalizado no núcleo do predicado, por meio, respectivamente, de prefixos pessoais ou prefixos relacionais. Consideramos, conforme argumentamos adiante, que são os argumentos marcados no núcleo do predicado por meio de prefixos pessoais que correspondem à noção de tópico frasal, enquanto os argumentos sintáticos $\mathrm{S} / \mathrm{A} / \mathrm{O}$, correspondem à noção de tópico discursivo, relacionado à importância deste elemento no discurso. Ao se introduzir novos elementos no discurso, o argumento sintático $\mathrm{S}$ ou os argumentos sintáticos A e O são obrigatórios. De outra maneira, somente um argumento sintático é expresso na sentença, sendo a sua ocorrência facultativa.

Consideramos, a partir da descrição de um sistema de hierarquia de pessoa em Avá-Canoeiro, que o Actor 1(2(3)), 2(3) ou 3 são mais tópicos quando agem sobre 3 (exemplos (7) a (11)); e o Undergoer 2(3) é mais tópico quando 1 age sobre ele(s) (exemplo (12)).

1(2(3)), 2(3) ou 3 agindo sobre 3

(7)

$$
\begin{array}{ll}
\mathbf{O} & \text { a-P } \\
\text { (kaj tõ) } & \text { a-juka } \\
\text { (macaco Foc) } & \text { 1-matar } \\
\text { '(macaco!) } & \text { eu } \\
\end{array}
$$

(8)

$$
\begin{array}{ll}
\mathbf{O} & \text { a-P } \\
\text { (kaj) } & \text { a-juka } \\
\text { (macaco) } & \text { 1-matar } \\
\text { '(macaco) } & \text { eu mato/matei'. }
\end{array}
$$

(9) $\mathbf{S} \quad \mathbf{s}-\mathbf{P}$

$$
\text { t } \mathrm{i} \text { tõ } \quad \mathbf{a} \text {-juka }
$$

1 FOC 1-matar

'eu, eu matei (ele)'

inerentemente mais tópicas do que as $3^{\text {as }}$ pessoas. Os participantes do ato de fala são também mais disponíveis na memória; por definição, se um ouvinte está à espera de um falante, o ouvinte deve ter um 'espaço aberto' para o falante. Existe um senso natural no qual os participantes de um ato de fala são geralmente tidos como 'mais importantes' ou o 'centro natural de interesse' em relação a uma $3^{\mathrm{a}} \mathrm{p}$. Então, sem observar qualquer contexto discursivo particular, a hierarquia pode ser tomada como uma hierarquia de topicalidade inerente" (Payne (1994: 316, tradução nossa) 
$(10)$
A
O
s-P
ne tõ ne l-etam-a
ele-japo tale
2 FOC $2=\mathrm{R}^{1}$-casa/aldeia-ARG
2 -fazer PROJ
'(...) tu vais fazer tua casa'

(11)

$$
\begin{array}{ll}
\mathbf{S} & \mathbf{s}-\mathbf{P} \\
\text { moj-a } & \mathbf{o}-\mathrm{t} \int \mathrm{u} \\
\text { cobra-ARG } & \text { 3-morder } \\
\text { 'a cobra mordeu (o rato)' }
\end{array}
$$

1(2(3)) agindo sobre 2(3)

$$
\begin{aligned}
& \text { o-P } \\
& \text { usu-kutuk } \\
& \text { 2O-furar } \\
& \text { 'furo-te (eu)' }
\end{aligned}
$$

No entanto, quando 3 age sobre 1(2(3)) ou 2(3) consideramos que não há expressão de tópico frasal, uma vez que o determinante do predicado é somente sinalizado em seu núcleo por meio de prefixo relacional (exemplos (13) a (15) abaixo), o que faz com que o predicado tenha leitura nominal.

3 agindo sobre 1(2(3)) ou 2(3)

$$
\begin{array}{ll}
\text { A } & \mathbf{O}=\mathbf{R}^{1}-\mathbf{P} \\
\text { Maria } & \mathrm{t} \mathrm{f} \text { Ø } \varnothing \text {-kutuk } \\
\text { Maria } & 1=\mathrm{R}^{1} \text {-furar } \\
\text { 'furar de } & \text { mim (por) Maria', 'Maria me furou (com agulha da injeção)' }
\end{array}
$$

$$
\begin{aligned}
& \mathbf{O}=\mathbf{R}^{1}-\mathbf{P} \quad \mathbf{A} \\
& \text { tfi Ø-kiti ekoj-a } \\
& 1=\mathrm{R}^{1} \text {-cortar DÊIT-ARG } \\
& \mathbf{O}=\mathbf{R}^{1}-\mathbf{P} \\
& \mathrm{t} \int \mathrm{i}=\varnothing \text {-juca-ema } \\
& 1=\mathrm{R}^{1} \text {-matar-PROIB }
\end{aligned}
$$$$
\text { 'o cortar de mim (por) esse (o facão)', 'esse (o facão) me corta/cortou' }
$$

'Não me mate!'7 (Granado 2005: 59)

\footnotetext{
${ }^{7}$ Por questões de polidez e confronto à face, é frequente a utilização, por parte dos AváCanoeiro (autodeterminados como Awã 'gente'), de $3^{\mathrm{a}} \mathrm{p}$. ao invés de $2^{\mathrm{a}} \mathrm{p}$. ao dirigirem a
} 
(16)

$$
\begin{array}{ll}
\text { A } & \mathbf{O}=\mathbf{R}^{1}-\mathbf{P} \\
\text { (Jawalitogo tõ) } & \mathrm{t} \int \mathrm{i}=\varnothing \text {-juka } \\
\text { (onça FOC) } & 1=\varnothing \text {-matar } \\
\text { '(onça!) o matar de mim' }
\end{array}
$$

$$
\begin{array}{ll}
\text { A } & \mathbf{O}=\mathbf{R}^{1}-\mathbf{P} \\
\text { (Jawalitogo) } & \mathrm{t} \int \mathrm{i}=\varnothing \text {-juka } \\
\text { (onça) } & 1=\varnothing-\text { matar } \\
\text { '(onça) o matar de mim' }
\end{array}
$$

Observamos, então, que a $3^{\text {a }}$ pessoa somente pode ser tópico frasal quando age sobre outra $3^{\text {a }}$ pessoa. Consideramos assim, que ela possui um escopo de topicalização reduzido. Além do mais, na língua Avá-Canoeiro, somente a terceira pessoa pode ser destopicalizada, quando um circunstante ao preceder o predicado engatilha o Modo Indicativo II.

Quando esse modo ocorre, o argumento S/O passa a ser sinalizado no núcleo do predicado por meio de prefixo relacional ao invés de prefixos pessoais, e o núcleo do predicado recebe um sufixo modal de modo Indicativo II. Apresentamos abaixo orações no modo Indicativo I (exemplos (18), (20) e (22)) em contraste com orações no modo Indicativo II (exemplos (19), (21) e (23)).

$$
\begin{array}{lll}
\mathbf{S} & \mathbf{s}-\mathbf{P} & \mathbf{S P} \\
\text { Ariel } & \mathbf{o} \text {-jus } & \text { kwa l-e } \\
\text { Ariel } & \text { 3-vir } & \text { longe } \mathrm{R}^{1} \text {-PosP } \\
\text { 'Ariel veio com } & \text { respeito à longe' }
\end{array}
$$

$$
\begin{array}{lll}
\text { SAdv } & \mathbf{R}^{2} \text {-P } & \text { SAdv } \\
\text { kasun } & \mathbf{i} \text {-tot-i } & \text { pe-pe } \\
\text { tarde } & \mathbf{R}^{2} \text {-ir-IND.II } & \text { DÊIT-Loc } \\
\text { 'de tarde } & \text { ele (o Paxe'o) } & \text { vai lá (naquele lugar)' }
\end{array}
$$

$$
\begin{array}{lll}
\text { S } & \text { s-P } & \text { SAdv } \\
\text { ae tõ } & \text { o-o } & \text { pe-pe } \\
\text { DÊIT tóp } & \text { 3-ir } & \text { DÊIT-LOC } \\
\text { 'ele (esse de quem } & \text { falamos) foi para lá' }
\end{array}
$$

$$
\begin{array}{lll}
\text { SAdv } & \text { A } & \mathbf{O}=\mathbf{R}^{1}-\mathbf{P} \\
\text { pe-pe } & \text { mail-a } & \text { awa- } \varnothing=\varnothing \text {-japiti-i } \\
\text { DÊIT-LOC FOC } & \text { Branco } & \text { pessoa-ARG R }{ }^{1} \text {-matar-IND.II } \\
\text { 'há tempos/lá longe, Branco matou muita gente' }
\end{array}
$$

palavra a um Maila 'não-indígena branco', diferentemente do uso de $2^{\mathrm{a}} \mathrm{p}$. ao interagirem com um outro Awã (Silva 2017, em preparação). 
$(22)$
A $\quad \mathbf{a}-\mathbf{P}$
$\mathrm{t} \int \mathrm{i}$ tõ $\quad \mathrm{a}-\mathrm{t} \int \mathrm{o}$
1 FOC 1-puxar
'eu puxei (o saco de arroz)'

$(23)$

\begin{tabular}{|c|c|c|}
\hline & a-P & $\mathbf{R}^{2}-\mathbf{P}$ \\
\hline ae tõ & ow-ев-а & $i-t \int o-w$ \\
\hline $\begin{array}{l}\text { DÊIT FOC } \\
\text { ['a:etõ }\end{array}$ & $\begin{array}{l}\text { 3CORR-C.C.-ir+GER } \\
\text { vg'we'ga: }\end{array}$ & $\begin{array}{l}\mathrm{R}^{2} \text {-puxar-IND.II } \\
\text { i't t o:we] }\end{array}$ \\
\hline
\end{tabular}

Observamos, desta forma, que a $3^{\mathrm{a}}$ pessoa, quando age sobre outra $3^{\mathrm{a}} \mathrm{p}$., é mais tópica em orações no modo indicativo I, sendo marcada no núcleo do predicado por meio de prefixo pessoal. Já no modo Indicativo II, por ter a contiguidade/não contiguidade de seus argumentos $\mathrm{S} / \mathrm{O}$ sinalizados no núcleo do predicado por meio de prefixos relacionais, é menos tópica.

A seguir tratamos da expressão de foco em Avá-Canoeiro do Tocantins.

\section{Foco em Avá Canoeiro do Tocantins e a partícula tõ}

Toral (1984/5: 5), foi o primeiro pesquisador a descrever parte do funcionamento da partícula tõ em Av.C-T. O autor (op. cit.) comenta que essa partícula possuiria diversos alomorfes - [-tõ-], [-otõ-], [-tõkõ-], etc. - e aparece, em seus dados, entre o prefixo de pessoa e a raiz do verbo. Para o autor, essa partícula "talvez funcione como enfatizador", sendo para os falantes de AváCanoeiro "provavelmente um recurso a dar mais ênfase às suas declarações para um ouvinte não familiarizado com a língua", uma vez que não foi observada a sua ocorrência na fala coloquial, na interação entre os Avá-Canoeiro e o pesquisador. De forma ilustrativa, o autor (Toral, 1984/5: 5) fornece os seguintes exemplos ${ }^{8}$ :

$$
\begin{array}{lll}
\text { A } & \text { a-P } & \text { SP } \\
\text { t } \int \mathrm{i} \text { tõ } & \text { a-u } & \text { i- } \varnothing \text { } \varnothing \text {-pupe } \\
\text { 1 FOC } & \text { 1-comer } & \text { água-ARG } \left.\text { R }^{1} \text {-PosP(dentro }\right) \\
\text { 'eu bebo } & \text { [a água de dentro]' }
\end{array}
$$

$$
\begin{array}{lll}
\text { S } & \text { SAdj } & \text { s-P } \\
\text { t } \int \text { i tõ } & \text { ko } & \text { a-wa-wak-i } \\
\text { 1 FOC } & \text { DÊIT } & \text { 1-REDUP-correr-IND.II } \\
\text { 'eu, aqui, eu corro muito' }
\end{array}
$$

\footnotetext{
${ }^{8}$ Mantemos a descrição fonética do autor com a adaptação para os símbolos do IPA mais recentes, bem como fornecemos a escrita fonológica com glosa e separação morfológica.
} 
(26) $\mathbf{S} \quad \mathbf{s}-\mathbf{P}$

ne tõ n ele-wa-wak-i

[ne tõ neKewéwekí]

2 FOC NEG 2-REDUP-correr-NEG

'tu não corres'

(27) $\mathbf{O} \quad \mathbf{a}-\mathbf{P}$

aw tõ a-mae-u

DÊIT FOC 1-coisa/animal-comer

'este, eu como (ingiro coisa)'

Borges (2006: 204-206) tece mais considerações acerca da distribuição e função desta partícula, considerada pela autora como uma marca de intensidade, 'intensivo' ('muito, mesmo'). Para Borges (op. cit.), esta marca ocorre em "final de enunciados ou em posição medial, após verbos intransitivos ativos e descritivos e também com verbos transitivos, bem como com a cópula eko $\sim i k o$ 'ser, estar"'. Ocorre também "após os clíticos $t / i=$ e $n i=$ e o demonstrativo ae= na formação dos pronomes pessoais de $1^{\mathrm{a}}, 2^{\mathrm{a}}$ e $3^{\mathrm{a}}$ pessoas do singular $t / \hat{i}=\tilde{t o}$, $n i=t \tilde{o}$ e $a e=t \tilde{o} "$, o que, segundo a autora, seria indicativo de um processo de gramaticalização. A partícula tõ poderia ainda ocorrer após nomes em primeira posição de enunciado, em função de sujeito e após a partícula ete ou иви em "verbos descritivos". Abaixo, reproduzimos alguns exemplos fornecidos pela autora para as situações supracitadas (mantemos a numeração original bem como a glosa da autora).

Verbos intransitivos ativos

(590a) Vintrans.

$n a=t \int i=i u-e j-i=t o ̃$

NEG-1SG=beber água-querer-NEG=PART.

'não estou com muita sede' (Borges, 2006: 204)

Verbos intransitivos descritivos

(591b) So

Vdescr.

akaj- $\varnothing \quad$ i-ete=tõ

cancão-CN 3-ser, estar gostoso=PART.

'O cancão é muito gostoso' (Borges, 2006: 204) 
Verbos transitivos

(593a) A Vtrans јашана- $\varnothing \quad$ о-евuг=tõ $\mathbf{P}$ cachorro-CN 3SG-trazer=PART. lagartixa-CN 'O cachorro trouxe a lagartixa' (Borges, 2006: 204)

Cópula eko iko 'ser, estar'

(594a) Sa tapira- $\varnothing=$ ete cópula anta-CN=PART 'A vaca está sentada mesmo' (Borges, 2006: 204)

Clíticos nos pronomes pessoais $t / i=e$ ni= e no demonstrativo ae $=$

$\mathrm{t} \int \mathrm{i}=\mathrm{tõ} \quad$ a-kir PRON.PESS.=PART. 1SG-dormir 'Eu dormi' (Borges, 2006: 190)

(556) ni=tõ ne=poij PRON.PESS. $=$ PART. $\quad 2 \mathrm{SG}=$ ser, estar gordo 'Você é gordo' (Borges, 2006: 190)

(558) ae=tõ $\quad \mathrm{t} f \mathrm{i}=\varnothing$-pikitr-a DEM=PART. $\quad 1$ POSS.SG=rel-irmã-CN 'Ela é minha irmã' (Borges, 2006: 190)

S nome em primeira posição

(595a) Sa amana- $\varnothing=$ tõ V chuva-CN-PART. 3sG-cair (chuva) 'Choveu demais' (Borges, 2006: 205) 
Após partícula "ete" e "uru"

(596b) So

\section{V}

maira- $\varnothing$

i-pot $\int \dot{i}=$ ete $=$ tõ

não-indígena-CN 3 -ser bravo, nervoso $=$ PART $=$ PART

'O não-indígena é muito bravo' (Borges, 2006: 205)

(596c) na-i-puku-usu=tõ

NEG-3-grande, comprido-AUM=PART.

'Não é muito comprido não' (Borges, 2006: 205)

Borges, por fim, propõe que essa partícula "se relacione a aspectos prosódicos e pragmáticos, como foco, enfatizando verbos, nomes e pronomes com os quais co-ocorre". Concorda, nesse sentido, com a suspeita inicial de Toral (1984/5), quanto a essa partícula ter função enfática.

A partir de novos dados, trazemos mais argumentos em favor da ideia de que essa partícula marca foco em Avá-Canoeiro do Tocantins, isto é, marca o elemento da sentença enfatizado-o, podendo o foco ser de natureza contrastiva. Até o momento não sabemos com precisão a origem dessa partícula, mas, muito provavelmente se deve a um desenvolvimento recente, anterior à migração de parte dos Avá-Canoeiro, no início de XIX, em direção à Ilha do Bananal, rio Araguaia. Seu desenvolvimento pode estar relacionado à alteração prosódica e estrutura informacional da língua, em decorrência da mudança na dinâmica de ocupação territorial, permeada por fugas e massacres. Conforme notam Toral (1984/5:5) e Borges (2006: 204), essa partícula não é encontrada no AváCanoeiro da variedade do rio Araguaia.

À luz de novos dados, observamos que essa partícula pode ser empregada tanto para focalizar argumentos (exemplos (28) a (34) $)^{9}$ quanto predicados de base nominais ou verbais (exemplos (35) a (40)) e expressões adverbiais (exemplos Erro! Fonte de referência não encontrada. a (43)), podendo ainda funcionar como foco contrastivo (exemplos (44) e (45)). Enquanto os argumentos focalizados com tõ ocorrem antes do predicado, os circunstantes e predicados focalizados não apresentam uma ordem preferencial.

Focalização de argumentos (pro)nominais Argumento $S$

$$
\begin{array}{ll}
\mathbf{S} & \mathbf{s}-\mathbf{P} \\
\text { ab-a tõ } & \text { o-ike } \\
\text { Sol-ARG FOC } & \text { 3-entrar } \\
\text { 'o Sol (já) entrou' (depois do pôr do sol) }
\end{array}
$$

\footnotetext{
${ }^{9}$ Até o momento não dispomos de dados em que $\mathrm{O}$ é focalizado por meio de tõ. Conforme veremos mais adiante, o Araweté, outra língua Tupí-Guaraní que dispõe de uma partícula de foco, $k u$, pode focalizar argumentos em função de objeto.
} 
$(29)$

\begin{tabular}{|c|c|c|c|}
\hline $\mathbf{S}$ & $s-P$ & $\mathbf{S}$ & $s-P$ \\
\hline ae tõ & o-kí & amin-a tõ & o-kís \\
\hline T FOC & 3-cair & chuva-ARG & FOc3-cair \\
\hline
\end{tabular}

Argumento A
A $\quad 0$
a-P
ne tõ ne l-etam-a
ele-japo tale
2 FOC $2=\mathrm{R}^{1}$-casa/aldeia-ARG 2 -fazer PROJ
'(...) tu vai fazer tua casa'

$(31)$
A $\quad \mathbf{a}-\mathbf{P} \quad \mathbf{O}$
t $\int$ i tõ a-itik $\varnothing$-pusu l-e
1 FOC 1-tirar $\mathrm{R}^{2}$-bolsa/cesto $\mathrm{R}^{1}$-POSP
'eu tirei o cesto (de cotia) (jogando-o fora)'

Sintagma nominal em oração equativa

(32)
SN
SN
iw-a tõ
t $\int$ i $\varnothing$-pilok-a
pau-ARG FOC $1=\mathrm{R}^{1}$-descascar-ARG
'o pau (de lenha), o meu descascar (dele)'

(33)
SN
SN
i-men-a tõ
ekoj-a
$\mathrm{R}^{2}$-marido-ARG FOC DÊIT-ARG
'o marido dela (é) esse'

Focalização de argumentos de base verbal
$\mathbf{S}=\mathbf{R}^{1}-\mathbf{P}$
na t $\int \mathrm{i} \varnothing$-u-i tõ
NEG $1=\mathrm{R}^{1}$-comer-NEG FOC
'não tenho (mais) fome' 
Focalização de predicados de base verbal

$\mathbf{O}=\mathbf{R}^{1}-\mathbf{P}$

na awa-Ø Ø-juka-j tõ

NEG pessoa-ARG ${ }^{1}$-matar-NEG FOC

'(esta planta) não mata gente' (em contraste com o fumo)

(36) $\quad \mathbf{a}-\mathbf{P}$

n a-u-j tõ

NEG 1-comer-NEG foc

'não comi (a rapadura)' (quando perguntado se a rapadura estava gostosa)

$\begin{array}{llll}\text { a-P } & \text { S } & \text { s-P } & \text { O } \\ \text { ubu-momew } & \text { Maria } & \text { t o-mo te } & \text { remédio } \\ \text { 13-contar } & \text { Maria } & \text { PROP 3-dar Lusiv remédio }\end{array}$

S

s-P

SAdv

Pãtfio no-kij-i tõ piaji-iw

Pãtfio NEG 3-morrer-NEG FOC noite/escuro-LOC

'nós falamos (então, para a) Maria, é para ela dar remédio (para a febre dele). Pãtfio, ele não morreu durante a noite (pois Parazinho ficou acordado, cuidando dele).'

(38)

$\left[\begin{array}{lll}\mathbf{s}-\mathbf{P} & \mathbf{S}\end{array}\right] \mathrm{O} \quad \mathrm{a}-\mathrm{P}$

o-ís tõ Sebastião $\quad \mathrm{n}$ a-kwa-j

3-vir FOC Sebastião NEG=1-saber-NEG

'ele veio, o Sebastião, eu não sei'

Focalização de predicados de base nominal

(39) (vocat) $\quad \mathbf{S}=\mathbf{R}^{1}-\mathbf{P}$

mat $\int a \quad$ na ne $\varnothing$-ai-te-j tõ

Mat $\int \mathrm{a} \quad$ NEG $2=\mathrm{R}^{1}$-ter.dor-GEN-NEG FOC

'Matfa, você não está sentindo dor?'

(40)

$\begin{array}{llll} & \mathbf{R}^{2} \text {-P } & & \mathbf{O}=\mathbf{R}^{1}-\mathbf{P} \\ \text { não } & \emptyset \text {-aime-te tõ } & \text { ae } & \mathrm{t} \int \mathrm{i} \emptyset \text {-kiti ne } \\ \text { não } & \mathrm{R}^{2} \text {-ser.afiado-GEN-FOC } & \text { DÊIT } & 1=\mathrm{R}^{1} \text {-cortar INTENC }\end{array}$

Não, (a lâmina) é muito afiada, assim (a faca) vai me cortar' 
Focalização de circunstâncias

$\begin{array}{ll}\text { SN } & \text { SAdv } \\ \text { koj-a } & \text { koem-i tõ } \\ \text { DÊIT-ARG } & \text { manhã-LOC FOC } \\ \text { 'esta manhã (a técnica de enfermagem vem)' }\end{array}$

$(42)$
SAdv
A
$\mathbf{O}=\mathbf{R}^{1}-\mathbf{P}$
pe-pe tõ
mail-a
awa-Ø Ø-apiti-j
DÊIT-LOC FOC
Branco-ARG
pessoa-matar.muito-IND.II
'há tempos/lá longe Branco matou muita gente'

(43)

$\begin{array}{llll}\text { S } & \text { s-P } & \text { SP } & \text { SAdv } \\ \text { awa- } \varnothing & \text { t o-pusai } & \text { pe Ø-pupe } & \text { ekwe tõ } \\ \text { gente-ARG } & \text { PROP 3-cantar } & \text { DÊIT R }{ }^{1} \text {-POSP } & \text { DÊIT FOC } \\ \text { eles, eles cantam (indo) dentro de lá (no poço onde se pesca) lá longe' }\end{array}$

Foco contrastivo

$$
\begin{array}{ll}
\text { a-P } & \text { a-P } \\
\text { e-u tía } & \text { a-u tale tõ } \\
\text { 2-comer PART } & \text { 1-comer PROJ FOC } \\
\text { 'coma, (pois) eu vou comer' }
\end{array}
$$
A
O
a-P
t $\int \mathrm{i}$ tõ
t-ata- $\varnothing$
a-mowe
1 FOC $\quad \mathrm{R}^{4}$-fogo-ARG
1-apagar
'fui eu que apaguei o fogo'

Borges(2006:205-206) consideraqueapartículatõestariase gramaticalizando na formação dos pronomes pessoais independentes e dos dêiticos. Em nossos dados, a partícula tõ não é, no entanto, uma marca obrigatória (vide exemplos (46) a (48) abaixo quanto aos pronomes independentes, e (49) a (51), quando aos dêiticos).

Pronomes pessoais independentes

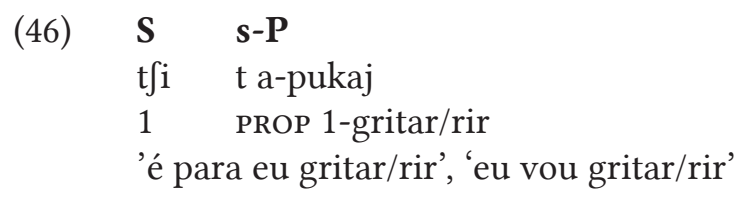


$(47)$

$$
\begin{array}{ll}
\mathbf{S} & \mathbf{s}-\mathbf{P} \\
\text { ne } & \text { ne } \varnothing \text {-katu-te } \\
2 & 2=\mathrm{R}^{1} \text {-bem/bom-GEN } \\
\text { 'você tem tua beleza verdadeira', 'você é bonito' }
\end{array}
$$

$$
\begin{array}{lll}
\text { A } & \text { a-P } & \text { O } \\
\text { pe } & \text { pe-jus } & \text { i-u-a } \\
\text { '23 } & \text { 23-vir } & \text { R}^{2} \text {-comer-ARG } \\
\text { ['pe } & \text { pe'zu:se } & \text {,i'u: }]
\end{array}
$$

'vocês, vocês venham para o comer (de arroz )'

\section{Dêiticos}

$$
\begin{array}{ll}
\mathbf{S} & \mathbf{O}=\mathbf{R}^{1}-\mathbf{P} \\
\text { ae } & \text { awa- } \varnothing ~ \varnothing \text {-juka } \\
\text { DÊIT } & \text { pessoa-ARG } \mathbf{R}^{1} \text {-matar } \\
\text { 'aquele (o peixe elétrico) mata gente' } &
\end{array}
$$

$$
\begin{array}{ll}
\mathbf{S} & \mathbf{s - P} \\
\text { ae } & \text { o-api-pai } \\
\text { DÊIT } & \text { 3-queimar-ASP(COMPL) }
\end{array}
$$

'aquele, ele se queimou todo, completamente'

$$
\begin{array}{ll}
\text { SN } & \text { SAdv } \\
\text { ew-a } & \varnothing \text {-puam-u } \\
\text { DÊIT-ARG } & \mathrm{R}^{2} \text {-em.pé-TRANSL } \\
\text { 'este, na qualidade de estando deitado' (foi pedido: ele está de pé', } \\
\text { logo após dizer que ele estava sentado) }
\end{array}
$$

A partícula tõ é uma partícula altamente produtiva que marca foco pragmaticamente motivado, a depender da intenção do falante em evidenciar um ou outro constituinte, marcando além de pronomes pessoais independentes e dêiticos, também predicados, circunstâncias e argumentos sintáticos. Ademais, considerar a partícula parte da forma fonológica dos pronomes seria aceitar a existência de um outro paradigma pessoal usado unicamente quando o referente pronominal fosse focalizado.

No âmbito da família Tupí-Guaraní há somente uma outra língua descrita que desenvolveu uma marca foco com função análoga à partícula tõ do Av.C-T, no entanto com desenvolvimento histórico diferente: a língua Araweté, subramo V (cf. Vieira \& Leite 1998; Solano 2009). Segundo Solano (2009: 379-386), a partícula $k u$ "delimita qual escopo da proposição deve ser tomado pelo ouvinte 
como relevante do ponto de vista do falante". Com isso, esta marca pode focalizar tanto o predicado, quanto o sujeito ou objeto da oração, bem como elementos circunstanciais (como sintagmas posposicionais, advérbios lexicais e orações no modo gerúndio), os quais, ao precederem o predicado, acionam o modo indicativo II.

Para a autora, quando a partícula $k u$ co-ocorre com elementos topicalizados, este "acentua o escopo do que é topicalizado". Nesse sentido, a autora sugere que, sendo o sujeito em orações SOV naturalmente mais tópico e podendo este ser focalizado por meio de $k u$, o uso desta partícula seria então "pragmaticamente condicionado, e que o fato de seguir também constituintes sujeito em primeira posição, pode significar que este também está em posição de tópico extrasentencial" (op. cit.), isto é, tópico discursivo. Segundo a autora, essa partícula em Araweté pode marcar também foco contrastivo. Reproduzimos abaixo alguns exemplos da autora para os contextos supracitados do Araweté, mantendo a numeração original, a título de ilustração.

Argumento sujeito

$$
\begin{array}{ll}
\text { A } & \mathbf{O}=\mathbf{R}^{1}-\mathbf{P} \\
\text { kume?e } k u & h e=\varnothing-p i-i k a \\
\text { homem FOC } & 1 \mathrm{R}^{1} \text {-pé-cortar } \\
\text { 'o homem (enfermeiro) cortou o meu pé' (Solano 2009: 381) }
\end{array}
$$

$$
\begin{array}{ll}
\mathbf{A} & \mathbf{O}=\mathbf{R}^{1}-\mathbf{P} \\
\text { he } k u & n e=\varnothing-u ? u \\
1 \text { FOC } & 2 \mathbf{R}^{1} \text {-morder }
\end{array}
$$

'Eu mordi você' (Solano 2009: 381)

Argumento objeto

$\begin{array}{llllll}\mathbf{O} & \mathbf{A} & \mathbf{a}-\mathbf{P} & \mathbf{R 3}-\mathbf{P} & \text { SAdv } & \\ \text { pehi ku } & \text { he } & \text { a-muji } & \text { te} \tilde{e} & \text { ka?arume } & a \text { a } \\ \text { cesto FOC } & 1 & \text { 1-fazer } & \text { 1CORR-estar.sentado } & \text { ontem } & \text { REIT } \\ \text { 'cesto eu estava fazendo ontem' (Solano 2009: 382) } & \end{array}$

$$
\begin{array}{lll}
\text { O } & \text { A } & \text { a-P } \\
\text { arapuha } k u & h e & a-? u \\
\text { veado FOC } & 1 & 1 \text {-comer } \\
\text { 'veado eu comi' (Solano 2009: 382) }
\end{array}
$$


Predicado

$$
\mathbf{O}=\mathbf{R}^{\mathbf{1}}-\mathbf{P}
$$

he Ø-nupĩ ku

$1 \mathrm{R}^{1}$-bater FOC

'ele me bateu' (Solano 2009: 381)

(1135)

$$
\begin{array}{ll}
\mathbf{a}-\mathbf{P} & \mathbf{O} \\
u \text {-pẽ } k u & \text { he Ø-jiete }
\end{array}
$$

3-quebrar FOC $1 \mathrm{R}^{1}$-machado

'ele quebrou meu machado' (Solano 2009: 381)

\section{Circunstâncias}

$$
\text { SAdv } \quad \mathbf{S}=\mathbf{R}^{1}-\mathbf{P}
$$

ka?aØ-iwe ku he Ø-tfe

mato $\mathrm{R}^{1}$-CI FOC $1 \mathrm{R}^{1}$-dormir

'dentro do mato, eu dormi' (Solano 2009: 383)

$\begin{array}{lll}\text { SAdv } & \text { O } & \text { a-P } \\ \text { hałwwe ku } & \text { akaju } & u \text {-tĩ } \\ \text { amanhã Foc } & \text { caju } & \text { 3-plantar }\end{array}$

'amanhã, eu plantarei caju' (Solano 2009: 383)

$$
\begin{array}{ll}
\text { SAdv } & \mathbf{S}=\mathbf{R}^{1} \text {-P } \\
\text { pẽ } n \text {-et } \int a k u & \text { he } r \text { - } u j i \\
23 \mathrm{R}^{1} \text {-ver FOC } & 1 \mathrm{R}^{1} \text {-voltar } \\
\text { 'eu voltei para ver vocês' (Solano 2009: 384) }
\end{array}
$$

Foco contrastivo

$$
\begin{array}{llllll}
\text { A } & \text { O } & \text { a-P } & \text { a-P } & & \text { A } \\
\text { Neura } k u & \text { at } \int a ? \dot{t} & u \text {-t } \int a \dot{t} & u \text {-t } \int a \dot{t} & \text { ja } & \text { Eliete } \\
\text { Neura FOC } & \text { açai } & \text { 3-amassar } & \text { 3-amassar } & \text { NEG } & \text { Eliete } \\
\text { 'foi a Neura que amassou o açai, não foi a Eliete' (Solano 2009: 385) }
\end{array}
$$
A
O $\quad \mathbf{a}-\mathbf{P}$
a-P
A
Kamaratiku iwahu u-?u u-?u já Ajajuru
Kamarati FoC mel 3-comer 3-comer NEG Ajajuru
'foi a Kamarati que comeu o mel, não foi o Ajajuru' (Solano 2009:386) 


\section{Considerações finais}

Descrevemos, para a língua Avá-Canoeiro, variedade do rio Tocantins, a expressão das categorias de pivô semântico, tópico e foco, buscando trazer contextos semelhantes de outras línguas da família Tupí-Guaraní.

O pivô semântico, para o Av.C-T, se realizaria na interface entre o nível semântico e o nível sintático, sendo previsto na semântica dos predicados, fazendo com que o Actor (argumentos S/A agente) engatilhe a marcação de correferencialidade em orações dependentes. O tópico frasal, em Avá-Canoeiro, seria expresso por meio dos prefixos pessoais, que são marcados no núcleo dos predicados. Uma vez que a marcação das pessoas do discurso nos predicados respeitam uma hierarquia de pessoa, notamos que a $3^{\mathrm{a}} \mathrm{p}$. é a pessoa menos tópica, sendo a única a se destopicalizar quando um elemento circunstancial é anteposto ao predicado. Caberiam aos argumentos sintáticos S/A/O a função de tópico discursivo, estando no nível extra-sentencial. Em Avá-Canoeiro, assim como em Araweté, a partícula de foco pode ser utilizada tanto para dar ênfase ou contrastar um constituinte ou oração, quanto, ao marcar um tópico discursivo, ressaltar a importância discursivo-pragmática deste constituinte ou oração. No entanto, enquanto em Araweté o argumento sintático $\mathrm{O}$, em primeira posição, pode ser marcado com essa partícula, em Avá-Canoeiro ainda não temos evidência dessa possibilidade.

\section{Referências Bibliográficas}

Borges, Mônica Veloso. 2006. Aspectos Fonológicos e Morfossintáticos da Língua Avá Canoeiro (Tupi-Guarani). (Tese de Doutorado), São Paulo, IEL-Unicamp.

Cabral, Ana Suelly A. C. Cabral; Silva, Ariel P. C. e Silva; Oliveira, Sanderson C. S. de; Sousa, Suseile, A.; Solano, Eliete de J. B. 2010. Análise contrastiva do fenômeno da correferencialidade em três línguas da família Tupí-Guaraní. In: Cabral, Ana Suelly A. C.; Rodrigues, Aryon D.; Duarte, Fábio B. (Org). Línguas e Culturas Tupí, v.2, pp. 95-104.

Cabral, Ana Suelly Arruda Câmara; Rodrigues, Aryon Dall'igna. 2003. Dicionário Asuriní do Tocantins-Português. Belém: UFPA/IFNOPAP; Brasília: UnB/IL/LALI.

Dixon, R. M. W. 1994. Ergativity. Cambridge: Cambridge University Press.

Foley, William A. e Van Valin Jr, Robert D. 1984. Functional syntax and universal grammar. Cambridge: Cambridge University Press.

Granado, Eliana. 2005. Fronteiras étnicas e fronteiras éticas furnas e a gestão da questão indígena. Dissertação (Mestrado). Rio de Janeiro, Centro de pesquisa e documentação de história contemporânea do brasil/ Programa de Pós graduação em História Política e Bens Culturais.

Monserrat, R. M. F., Soares, M. F. 1983. Hierarquia referencial em línguas Tupí. Ensaios de Lingüística 9:164-187. 
Payne, D. 1994. The Tupí-Guaraní inverse. In Fox, B.; Hopper, P.. Voice, form and function. Amsterdam: John Benjamins Publishing, pp. 313-340.

Rodrigues, Aryon D. 1985. Relações Internas na Família Linguística Tupi-Guarani. Revista de Antropologia 27:33-53.

Rodrigues, Aryon Dall'Igna; Cabral, Ana Suelly Arruda Câmara. 2002. Revendo a classificação interna da família Tupí-Guaraní. In: Cabral, A. S. A. C., Rodrigues, A. D. (Orgs.). Línguas indígenas brasileiras: fonologia, gramática e história. Tomo I. Belém: UFPA, pp. 327-337.

Silva, Ariel Pheula do Couto e. 2015. Elementos de fonologia, morfossintaxe e sintaxe da língua Avá-Canoeiro. Dissertação (Mestrado em Linguística), Brasília, UnB/IL/ LIP/PPGL.

. 2017. Uma análise da interação Awã (Avá-Canoeiro) e Maila (não-indígena branco): quando o Tu é um Outro. (em preparação).

Solano, Eliete de Jesus Bararuá. 2009. Descrição Gramatical da Língua Araweté. Tese de Doutorado. Brasília: UnB/IL/LIP/PPGL.

Toral, André Amaral. 1984/1985. Os índios negros ou os Carijó de Goiás: a história dos Avá-Canoeiro. Revista de Antropologia 27-28:287-325, São Paulo, USP.

. Vocabulário Avá-Canoeiro. s/d. (mimeo).

Vieira, Márcia M. D. 2015. A Manifestação de Tópico e Foco em Línguas da Família Tupí-Guaraní. D.E.L.T.A.30 (especial):659-683. Disponível em: <http://www. scielo.br/scielo.php?script=sci_arttext\&pid=S0102-44502014000300659\&lng=en $\& t \operatorname{lng}=\mathrm{pt}>$, Acesso em: ago./2017.

Vieira, Márcia M. D. \& Leite, Yonne F.. 1998. Observações Preliminares sobre a Língua Araweté. Moara-Revista dos Cursos de Pós-Graduação em Letras 9: 7-31, Belém, UFPA. 